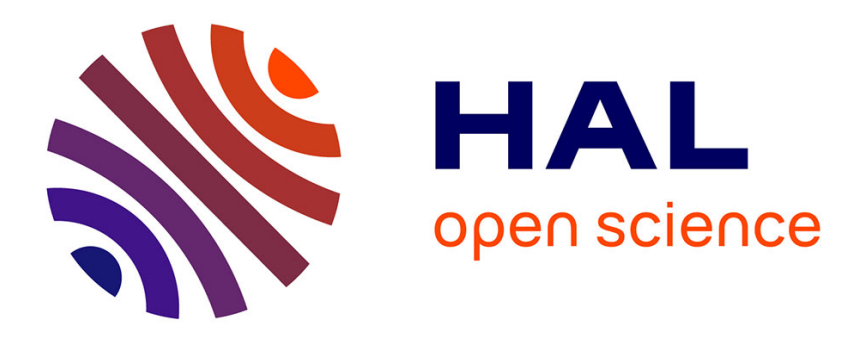

\title{
THE PERCOLATION APPROACH TO NUCLEUS BREAK-UP \\ X. Campi
}

\section{To cite this version:}

X. Campi. THE PERCOLATION APPROACH TO NUCLEUS BREAK-UP. International Conference on Heavy Ion Nuclear Collisions in the Fermi Energy Domain Hicofed 86, 1986, Caen, France. pp.C4-419-C4-422, 10.1051/jphyscol:1986449 . jpa-00225815

\section{HAL Id: jpa-00225815 https://hal.science/jpa-00225815}

Submitted on 1 Jan 1986

HAL is a multi-disciplinary open access archive for the deposit and dissemination of scientific research documents, whether they are published or not. The documents may come from teaching and research institutions in France or abroad, or from public or private research centers.
L'archive ouverte pluridisciplinaire HAL, est destinée au dépôt et à la diffusion de documents scientifiques de niveau recherche, publiés ou non, émanant des établissements d'enseignement et de recherche français ou étrangers, des laboratoires publics ou privés. 


\title{
THE PERCOLATION APPROACH TO NUCLEUS BREAK-UP
}

\author{
X. CAMPI \\ Division de Physique Theorique*, Institut de Physique Nucléaire, \\ F-91406 Orsay Cedex, France
}

\begin{abstract}
Résumé
La rupture des noyaux dans les collisions violentes est étudiée comme un phénomène critique. On montre comment on peut déterminer. plusieurs exposants critiques à partir des données expérimentales sur la distribution de masse des fragments et on les compare avec les prédictions du modèle de la percolation.
\end{abstract}

\begin{abstract}
Nucleus break up in violent collisions is studied as a critical phenomenon. We show how to determine various critical exponents from experimental data on mass distributions. A comparison is made with the predictions of percolation theory.
\end{abstract}

The problem of the size distribution of nuclear fragments produced in energetic collisions has received considerable interest in the last years $/ 1-8 /$. However we can still address the following basic question : Do the size distribution reflects some specific properties of nuclear matter under extreme conditions or just follows the same "universal" laws as many other disordered systems? In other words, do the nuclear specificities (nuclear and Coulomb interactions, collision dynamics...) play a crucial role or not ?

In this contribution we review some of the results obtained exploring the second hypothesis, with special emphasis on the percolation approach. The theory of percolation models deals with clusters or randomly occupied sites in a lattice. Percolation models are governed by a single parameter $p$ representing the density or fraction of sites that are occupied or links that are active. Above

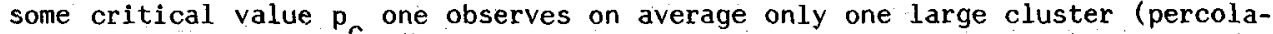
tion or infinite cluster) occupying most of the active sites, while for $p<p$, many small clusters are present. The transition from a "percolating" to a "non percolating" state is a kind of phase transition one can characterize by a set of critical exponents, like in thermal phase transitions.

The works of Refs./5-8/ are practical realizations of the percolation concept applied to nuclear reactions. The principal characteristics of the various models are summarized in Table 1 , where is indicated the type of percolation used (site or bond), the lattice, the dimension of the space in which the lattice is defined, the percolation threshold and the coordination number (maximum number of bonds per site). We see on Table 1 that the predicted percolation threshold depends strongly on the details of the model. The same problem occurs in thermal phase transitions with the critical temperature $/ 3 /$. What is the right space and lattice, the right linkage condition to describe a nucleus in a collision ? Do we need extra parameters, like a temperature ? It seems now very difficult to answer at these basic questions. Fortunately we can approach the problem in a

\footnotetext{
"Laboratoire essocie au C.N.R.S.
} 
much simpler way. If nuclear multifragmentation has something to do with a critical phenomenon, then we can look at critical exponents.

\begin{tabular}{|l|c|c|c|c|c|}
\hline Mode1/Ref & Type & Lattice & $\mathrm{d}$ & $\mathrm{z}$ & $\mathrm{p}_{\mathrm{c}}$ \\
\hline CDL84 $/ 5 /$ & Bond & Line & 1 & 2 & 1.0 \\
BDMP85 /6/ & Bond & Cubic & 3 & 6 & 0.31 \\
CD85 $/ 7 /$ & $\begin{array}{c}\text { Site } \\
\text { Continuous } \\
\text { phase space } \\
\text { square/ } \\
\text { cubic }\end{array}$ & $3+3$ & $2-3$ & $4-6$ & $0.59-0.31$ \\
\hline
\end{tabular}

Table 1 - Principal characteristics of nuclear percolation models

Despite large differences in the predicted value of $p_{c}$, the various nuclear percolation models (in 3-dimensions) predict rather similar cluster size distributions. This is because these models belong to the same class of universality and so have approximately the same critical exponents. We restrict in what follows to quantities associated with cluster size distributions that can be defined in percolation as well as in thermal phase critical models of the liquid-gas type, just replacing $\left(p-p_{f}\right)$ by $(T-T)$. We examine the following: a) near the critical point $p=p_{c}$ the mulfiplicity of light clusters of size A behaves like /10/,

$$
n(A, p) \sim A^{-\tau} f\left(\left(p-p_{c}\right) A^{\sigma}\right)
$$

where $\tau$ and $\sigma$ are two critical exponents and $f(0)=1$. In 3-dimension percolation, $\tau=2.2$ (see Table 2). For the thermal liquid-gas model at $T=T_{c}$ $n(A, T) \sim A^{-\tau}$, with $\tau=7 / 3$, a very close value $/ 3 \%$. At $p<p_{c}$ and $p>p_{c}^{c}$ the asymptotic decay of cluster numbers is given by

\begin{tabular}{|c|c|c|}
\cline { 2 - 3 } \multicolumn{1}{c|}{} & Perc. & L-G \\
\hline$\tau$ & 2.2 & $7 / 3$ \\
$\sigma$ & 0.45 & $2 / 3$ \\
$\xi^{ \pm}\{$ & 1 & 1 \\
$\gamma$ & $2 / 3$ & $2 / 3$ \\
$\beta$ & 1.8 & 1 \\
\hline
\end{tabular}

Table 2 - Critical exponents defined by eqs $(1-5)$ in percolation and thermal liquid-gas phase transitions. From refs. $/ 3 /$ and /10/

$$
n(A, p) \sim \exp \left(-A^{\xi^{ \pm}}\right)
$$

with different values above and below the threshold, but identical for percolation and liquidgas. We see that with these two exponents it is impossible to distinguish between the two theories. However, the experimental value of $\tau$ has been studied in great detail on the basis of inclusive data $/ 11 /$. This data is even more difficult to interpret because it reflects the superposition of events with a wide range of values of $p$, only a few corresponding to $p=p$ (or to $T=T_{c}$ ). In view of Eqs. (2) and (3) we can say that the "effective" exponent obtained fitting inclusive data is $\tau_{\text {eff }} \geqslant \tau$. The equality holds only at $p=p_{0}:(T=T)$. In order to study the $p$ or $T$ dependence of $\mathcal{C}$ we need data from exclusive experiments, event by event. Still another problem arises : How to correlate the events with $p$ or $T$ ? One possibility is to assume that the number of $Z=1$ particles released in a reaction is an increasing function of $T$ or $1-p / 12 /$. Figure 1 shows an attempt to analyze data on energetic $\mathrm{Au}+(\mathrm{BrAg})$ reactions from $\mathrm{Ref} . / 13 /$. We get the expected evolution of $\tau$.f. According to this analysis, the critical point should arrive for events with about 10 to $15 \mathrm{Z}=1$ particles. Remark that $\tau<2$ is incompatible with phase transition theories.

b) The moments of the light cluster size distributions

$$
S_{k}=\sum A^{k} n(A, p) / \sum A n(A, p) \propto\left|p-p_{c}\right|^{-\varphi_{k}}
$$

define two new exponents for $k=2$ and $k=3$. The sums run over all "finite" size clusters (the largest one excluded). The exponent for $k=2$ is usually called $\gamma$ (see table 2). Assuming the scaling relation (1), then /10/ 


$$
\varphi_{k}=(\tau-1-k) / \sigma
$$

If this relation is still valid in our finite system, then $\mathrm{S}_{3} / \mathrm{S}_{2}$ behaves like $(\tau-4) /(\tau-3)$ near the critical point, or also like $1+1 / \sigma \cdot \gamma^{3}$. 2 of course in nuclei. (3) will never diverge, just take much larger values near $p=p_{c}$. We can check this point plotting $\ell n\left(S_{3}\right)$ versus $\ell n\left(S_{2}\right)$. This procedure has also the advantage to eliminate the unknown dependence on $p-p$. The slope of the line gives the ratio of the critical exponents. The result for the data of Ref./13/ is plotted in Fig.2. Each point represents one event. A least square fit gives $\sigma \cdot \gamma \approx 0.82$ which is surprisingly close to the prediction of percolation $\left(\sigma . \gamma=0.81^{2}\right.$ for percolation and 0.66 for liquid-gas). Alternatively also we deduce from $(4) \quad \tau \approx 2.16$.

c) Finally, we consider the "critical" behaviour of the size of the largest cluster produced by event

$$
P(p)-\left(p-p_{c}\right)^{\beta}
$$

Again we eliminate the unknown $p-p_{c}$ dependence comparing $\ln (P)$ versus $\ell n\left(S_{2}\right)$, getting - $\beta / \gamma$. This ratio seems very interesting because $\gamma / \beta \sim 4.5$ in percolation but only $\sim 2.0$ in liquid-gas. Preliminary analysis of the data of Ref./13/ seems to favour a large value for $\gamma / \beta$

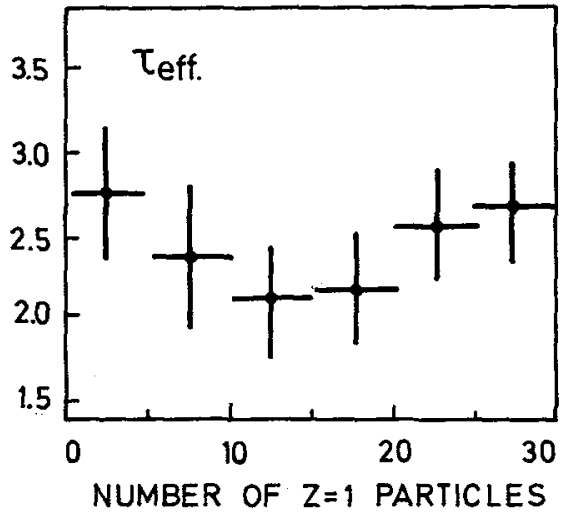

Fig. 1 - Effective slope parameter $\sigma \sim Z^{-\tau \text { eff }}$ resulting from the fit of $2<Z<14$ projectile fragments of the reaction $\mathrm{Au}+(\mathrm{BrAg})$ plotted against the number of $Z=1$ particles.

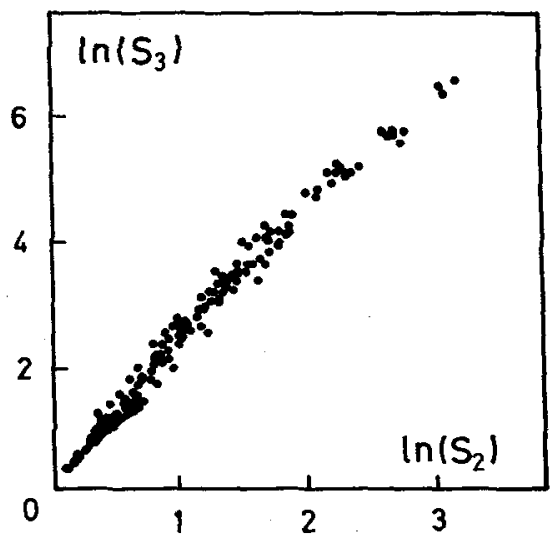

Fig.2 - Determination of $1 / \sigma \cdot \gamma$ from eqs. (3) and (4). Each point represents one event of the reaction $\mathrm{Au}+(\mathrm{BrAg})$.

We believe that with better statistics the exponents $\tau, \sigma, \beta$ and $\gamma$ can be determined very accurately. However, there is still another open problem. For an infinite system, the critical exponents depend only on the dimensionality of the order parameter and the dimensionality of the physical space. What is the size and lattice dependence in a finite system ? This question is still very controversial. Some numerical tests on percolation seem to indicate that this dependence is rather weak /14/. If this is confirmed, then we could give a definite answer to the basic question raised at the beginning.

/1/ G. Bertsch and D. Mundinger, Phys. Rev. C17 (1977) 1646.

121 J.P. Bondorf et al., Phys. Lett. 150B (1985) 57 ;

D.H.E. Gross et al., Phys. Rev. Lett. 56 (1986) 1544.

13/ R.W. Munich et al., Phys. Lett. 118B (1982) 458 ;

P.J. Siemens, Nature 305 (1983) 410 ;

A.L. Goodmann et al., Phys. Rev. C30 (1984) 851. 
14/ J. Aichelin and H. Hüfner, Phys. Lett. 136B (1984) 15.

15/ X. Campi et al., Nucl. Phys. A428 (1984) 327.

16/ W. Bauer et al., Phys. Lett. 1508 (1985) 53.

17/ X. Campi and 3. Desbois, CSI Rep. 85-10 ; Cont. Bormio Conf. 85, Supp. 47 Ric. Sc. Ed. Perm. p. 495 ; Phase Space Approach to Nucl. Dynamics, ed. Di Toro (World Sci., Singapore, 1986).

/8/ T.S. Biro, J. Knoll and J. Richert, Preprint GSI, Darmstadt, 1986.

19/ H.W. Barz et al., Phys. Lett. 169B (1986) 318.

$110 /$ D. Stauffer, in "Introduction to Percolation Theory", Taylor and Francis Ed. 1985.

111/ A.D. Panagiotou et al., Phys. Rev. C31 (1985) 55.

112 J. Aichelin and X. Campi, MPI Heidelberg, preprint.

/13/ C.J. Waddington and P.S. Freier, Phys. Rev. C31 ( 1985) 888.

114 / J.P. Clerc et al., J. Phys. Lett. 42 (1981) 249. 